\title{
Treinamento de força diminui os sintomas depressivos e melhora a qualidade de vida relacionada a saúde em idosas
}

CDD. 20.ed. 796.018

796.073

http://dx.doi.org/10.1590/1807-55092015000200189

\author{
Letícia BRUNONI* \\ Felipe Barreto SCHUCH ${ }^{* * * * * *}$ \\ Caroline Pieta DIAS** \\ Luis Fernando Martins KRUEL ${ }^{* *}$ \\ Carlos Leandro TIGGEMANN****
}

*Faculdade da Serra Gaúcha.

${ }^{* *}$ Escola de Educação Física, Universidade

Federal do Rio Grande do Sul.

***Hospital das Clínicas de Porto Alegre.

\section{Resumo}

0 objetivo do presente estudo foi de avaliar os efeitos do treinamento de força, com prescrição baseada na percepção de esforço, nos sintomas depressivos e na Qualidade de Vida Relacionada à Saúde (OVRS) de idosas. Participaram do estudo 24 idosas (idade de $65,6 \pm 4,5$ anos), submetidas a um treinamento de força duas vezes por semana, durante 12 semanas com intensidade prescrita através da escala de percepção de esforço de BORG. Utilizou-se a escala de depressão geriátrica (EDG) de para rastreio de depressão e avaliação dos sintomas depressivos. Para avaliação da QVRS, utilizou-se o Questionário de Qualidade de Vida Short Form-36 (SF-36). Para comparação das variáveis pré e pós-treinamento foi utilizado o teste de Wilcoxon. Foi encontrada uma redução dos sintomas depressivos, bem como da frequência de idosas com pontuação indicativa de episódio depressivo atual, após o treinamento de força. Para a QVRS, foram encontradas melhoras nas subescalas de capacidade funcional, estado geral da saúde, vitalidade e saúde mental. 0 treinamento de força, baseado na percepção de esforço, foi um método efetivo para uma redução nos sintomas de depressivos das participantes bem como para melhora da QVRS relacionada à saúde nas seguintes subescalas do SF-36: vitalidade, capacidade funcional, estado geral da saúde e saúde mental em idosas.

PalavRAS-Chave: Depressão; Envelhecimento; Exercício; Mulheres; Qualidade de vida; Treinamento de força.

\section{Introdução}

O envelhecimento é um fenômeno altamente complexo e comum a todos os membros de uma determinada espécie, progressivo, envolvendo mecanismos deletérios que afetam a capacidade de desempenhar um grande número de funçôes ${ }^{1}$. $\mathrm{O}$ aumento da população idosa está associado à prevalência elevada de doenças crônico-degenerativas, dentre elas aquelas que comprometem o funcionamento do sistema nervoso central, particularmente a depressão ${ }^{2}$. A depressão é um transtorno crônico, de curso prevalente ${ }^{3}$. Dentre os sintomas da depressão, destaca-se a necessidade da presença da tristeza, todos os dias ou na maior parte dos dias, ou a perda do prazer e do interesse em atividades que antes eram prazerosas ${ }^{4}$. A depressão possui um grande impacto negativo na Qualidade de Vida Relacionada a Saúde
(QVRS), afetando diretamente diversos aspectos da vida, dentre os quais pode-se salientar prejuízos sociais, afetivos e laborais 5 .

Por outro lado a participação em programas de exercício físico pode retardar declínios funcionais bem como diminuir os sintomas depressivos, o risco do desenvolvimento de episódios depressivos ${ }^{6}$ e melhorar a QVRS 7 . A literatura, entretanto, não é conclusiva a respeito da forma de prescrição mais adequada para se chegar a este efeito. Segundo Staton e Happell ${ }^{8}$, de 30 a 40 minutos de exercício físico aeróbico, realizados com uma frequência mínima de três vezes na semana, com a intensidade preferida pelo paciente seriam suficientes para obter uma redução significativa nos sintomas depressivos. Em relação ao treino de força, alguns estudos em 
idosos sugerem que 60-65\% de uma repetição máxima (1 RM) são eficazes para reduzir os sintomas depressivos ${ }^{8-9}$. Entretanto, nenhum estudo avaliou os efeitos do treinamento de força prescrito através da percepção de esforço visando à redução dos sintomas depressivos e da melhora da QVRS em idosas.

A prescrição da carga feita através da percepção de esforço é uma alternativa eficaz para promover aumentos dos níveis de força em idosos ${ }^{10}$. Como vantagens em relação à prescrição tradicional, esse método pode ser uma alternativa rápida e que produz menos desconforto, bem como respostas cardiovasculares mais amenas quando comparado à prescrição através do teste de uma repetição máxima $^{11-12}$. Além disso, estudos tem identificado similares ganhos de força máxima em grupos de homens jovens que realizam treinamentos em sérias

\section{Método}

\section{Desenho do estudo}

Esta pesquisa caracteriza-se como um estudo experimental longitudinal com amostra por conveniência ${ }^{15}$. Este estudo foi aprovado pelo Comitê de Ética em Pesquisa da Universidade Federal do Rio Grande do Sul (registro n. 22108). O estudo caracteriza-se por uma intervenção com treinamento de força com duração de 12 semanas em mulheres idosas, tendo como principais variáveis dependentes a avaliação dos sintomas depressivos e melhora da QVRS. Previamente ao início do treinamento todas as idosas foram familiarizadas com os equipamentos, bem como a realização de testes (semana menos quatro) e reteste (semana zero) da força máxima.

\section{Amostra}

A amostra foi recrutada no período de janeiro a março de 2012, sendo constituída de 24 mulheres acima de 60 anos da cidade de Caxias do Sul/RS. As características da amostra são apresentadas na TABELA 1. Para serem incluídas na amostra, as participantes não poderiam praticar exercícios físicos regulares (frequência maior do que uma sessão semanal) nem ter participado de treinamento de força nos últimos 12 meses, embora 83,3\% fossem classificadas como fisicamente ativas pelo Questionário Internacional de Atividade Física (IPAQ ${ }^{16}$. Além disso, as mesmas não poderiam apresentar histórico de doenças até a exaustão (repetiçôes máximas), quando comparados com grupos não usam esta estratégia ${ }^{13}$. Em idosos, apesar da ausência de estudos que avaliem as adaptaçóes neurais, incrementos na força máxima, aumentos significativos na espessura muscular também tem se mostrado táo efetivos por meio do uso de esforços submáximos (uso da percepção de esforço), quanto com o uso de repetiçôes máximas ${ }^{14}$.

Diante do exposto, a hipótese do estudo seria que a prescrição do treinamento de força por meio da percepção de esforço pode ser utilizada como uma estratégia eficaz para o treinamento de força objetivando a redução dos sintomas depressivos e a melhora da QVRS. Portanto, o objetivo do presente estudo foi de avaliar o efeito do treinamento de força com intensidade prescrita por meio da percepção de esforço em idosas na melhora dos sintomas depressivos e melhora da QVRS.

cardiovasculares (a exceção de hipertensão arterial controlada por medicamento), endócrinas, metabólicas, neuromusculares e articulares, justificados por meio de atestado clínico médico. Em uma escala de zero a 10 quanto a auto avaliaçấo de sua saúde, o grupo apresentou uma média de $8,21 \pm 1,06$ pontos.

TABELA 1 - Média, desvio-padrão e valores mínimos e máximos das características da amostra.

\begin{tabular}{lcc}
\hline & Média \pm DP & Mínimo/máximo \\
\hline Idade (anos) & $65,6 \pm 4,5$ & $60-75$ \\
Massa corporal $(\mathrm{kg})$ & $65,1 \pm 8,5$ & $50,0-82,8$ \\
Estatura $(\mathrm{cm})$ & $156,1 \pm 4,8$ & $146-166$ \\
IMC $\left(\mathrm{kg} / \mathrm{m}^{2}\right)$ & $26,7 \pm 3,3$ & $21,6-33,4$ \\
\hline
\end{tabular}

\section{Protocolo experimental}

As idosas foram submetidas a um protocolo de treinamento de força com cargas selecionadas por meio da percepção de esforço. A carga utilizada iniciou na intensidade correspondente ao esforço $13 \pm 1$ da escala RPE de BorG ${ }^{17}$, nas duas primeiras semanas, $14 \pm 1$ nas semanas 3 e $4,15 \pm 1$ nas semanas 5 e 6 , $16 \pm$ nas semanas 7 e $8,17 \pm 1$ nas semanas 9 e 10 e $18 \pm 1$ nas duas últimas semanas ${ }^{18-20}$. A cada sessão de treinamento, ao final da última série de cada exercício, a percepção de esforço era avaliada, e estando a mesma no valor estipulado para a sessáo a mesma era mantida para a sessão seguinte, e caso contrário, aumentada 
ou diminuída em $5-10 \%$ da carga. Ao final do treinamento, com base nos valores do teste de uma repetiçấo máxima (1 RM) avaliados, identificou-se que a carga utilizada correspondeu entre 45,69 $\pm 3,73$ a 73,45 \pm $7,21 \% 1 \mathrm{RM}$. O treinamento foi realizado durante 12 semanas com frequência de duas vezes por semana, sendo finalizado com uma aderência de treinamento superior a 98\%. Cada sessáo de treinamento foi composta por um breve aquecimento aeróbico $(5 \mathrm{~min})$ e articular. Posteriormente eram realizados os exercícios principais na seguinte ordem: pressão de pernas, supino plano, flexão de joelhos, puxada frontal e extensão de joelhos, com um intervalo de descanso entre cada série e exercícios de aproximadamente 1,5 minutos, em um ritmo de execução de dois segundos para cada fase. A parte final foi composta de uma série de alongamentos. $\mathrm{O}$ treinamento de força teve progressão de volume e intensidade periodizados de forma linear. $\mathrm{O}$ treinamento foi aplicado e monitorado por indivíduos devidamente instruídos e preparados para atuarem no experimento.

\section{Protocolos de avaliação}

O nível de atividade física habitual foi avaliado previamente ao protocolo experimental por meio do IPAQ (versão curta) ${ }^{16}$ com objetivo de caracterizar a amostra. A classificação dos sujeitos foi realizada por meio da soma dos minutos de atividades físicas realizadas em uma semana habitual, conforme critérios estabelecidos pelos protocolos de aplicação do questionário. Para a avaliação da força máxima, o teste de 1 RM foi utilizado em todos os cinco exercícios. $\mathrm{O}$ teste consiste na realizaçáo de apenas uma repetiçáo com o máximo de carga possível dentro de um padrão de execução, seguindo as recomendaçóes de Brown e WeIR $^{21}$, sendo avaliada nas semanas -4, 0, 4, 8 e 12.

\section{Resultados}

Os resultados do treinamento de força indicam aumentos significativos $(\mathrm{p}<0,001)$ em todos os exercícios avaliados, com incrementos que variaram de $16,55 \pm 5,82$ a $56,35 \pm 14,57 \%$ (TABELA 2).

Foi encontrada uma reduçáo nos sintomas depressivos do período pré-treinamento para o pós-treinamento $(9,5 \pm 4,25$ versus $6,00 \pm 6,75$; $\mathrm{p}<0,001)$. Analisando os resultados baseados nos pontos de corte definidos para episodio depressivo atual, o resultado da EDG apontou que 45,8\% das idosas apresentavam pontuação igual ou superior a
Para a avaliação dos sintomas depressivos e da QVRS à saúde foram utilizados os seguintes instrumentos: Escala de Depressão Geriátrica (EDG) e questionário de avaliaçáo da qualidade de vida SF36, ambos aplicados em forma de entrevista individual. A EDG é um dos instrumentos mais utilizados no rastreio de depressão e avaliação de sintomas depressivos em pacientes idosos. $\mathrm{Na}$ sua versão de 30 itens, uma pontuaçáo igual ou maior que 11 é indicativa de provável episódio depressivo atual. $\mathrm{O}$ instrumento tem sensibilidade e especificidade satisfatórias e foi traduzido para o português. Uma pontuação de 11 ou mais é indicativa de provável episodio depressivo atual ${ }^{22}$. O segundo questionário, o SF-36 é um instrumento de avaliaçáo da QVRS. É considerado um questionário multidimensional formado por 36 itens, composto por oito componentes: capacidade funcional, aspectos físicos, dor, estado geral da saúde, vitalidade, aspectos sociais, aspectos emocionais e saúde mental. Apresenta um escore para cada componente que varia de zero a 100 , no qual zero corresponde ao pior estado e 100 ao melhor estado ${ }^{23}$.

\section{Análise estatística}

A normalidade e homogeneidade dos dados foram testadas por meio dos testes de Shapiro-Wilk e Levene respectivamente. Os dados são apresentados em média \pm desvio padrão e frequências. A comparação da força máxima foi realizada por meio do teste t pareado. Para comparação das variáveis dependentes de pré e pós-treinamento foi utilizado o teste de Wilcoxon e teste do chi-quadrado. O nível de significância adotado foi $\alpha \leq 0,05$. Os dados foram analisados no "software" SPSS, versão 18.0.

11 no período pré-treinamento, sendo que após o treinamento, apenas $16,7 \%$ apresentaram pontuaçáo igual ou superior a 11 pontos. A distribuição dos participantes em cada faixa de sintoma pode ser vista na TABELA 3.

Em relação à QVRS, foram observadas diferenças significativas após o período de treinamento nos componentes: capacidade funcional, estado geral de saúde, vitalidade e saúde mental. Para os demais aspectos não foram encontras diferenças do período pré para o pós-treinamento (TABELA 4). 
TABELA 2 - Valores absolutos de média e desvio padrão dos testes de força máxima (1 RM = uma repetição máxima) nos diferentes exercícios avaliados, nos momentos pré e pós intervenção $(\mathrm{p}<0,05)$.

\begin{tabular}{lccc}
\hline Exercício - 1 RM $(\mathbf{k g})$ & Pré-treinamento & Pós-treinamento & p \\
\hline Extensão de joelhos & $24,50 \pm 3,08$ & $28,48 \pm 3,30$ & $<0,001$ \\
Flexão de joelhos & $14,31 \pm 3,09$ & $18,56 \pm 2,28$ & $<0,001$ \\
Pressão de pernas & $61,33 \pm 10,46$ & $95,75 \pm 18,33$ & $<0,001$ \\
Supino plano & $26,63 \pm 5,49$ & $35,71 \pm 5,28$ & $<0,001$ \\
Remada baixa & $41,08 \pm 5,72$ & $49,19 \pm 5,27$ & $<0,001$ \\
\hline
\end{tabular}

TABELA 3 - Frequência absoluta e relativa da escala de depressão geriátrica (EDG) pré e pós-treinamento no grupo avaliado.

\begin{tabular}{lcccc}
\hline & \multicolumn{2}{c}{ Pré-treinamento } & \multicolumn{2}{c}{ Pós-treinamento } \\
\hline Pontuação & $\mathrm{n}$ & $\%$ & $\mathrm{n}$ & $\%$ \\
\hline$\geq 6$ & 2 & 8 & 12 & 48 \\
$<6$ e $\geq 8$ & 5 & 20 & 3 & 12 \\
$<8$ e $\geq 10$ & 7 & 28 & 5 & 20 \\
$<10$ e $\geq 12$ & 6 & 24 & 0 & 0 \\
$\leq 12$ & 5 & 20 & 4 & 16 \\
\hline
\end{tabular}

Dados apresentados por média e desvio padrão;

$p \leq 0,05$ indica diferença significativa.

TABELA 4 -QVRS pré e pós-treinamento. Dados apresentados por media e desvio padrão. $\mathrm{p} \leq$ o,05 indica diferença significativa.

\begin{tabular}{lccc}
\hline & Pré-treinamento & Pós-treinamento & p \\
\hline Capacidade funcional & $77,3 \pm 18,1$ & $86,0 \pm 11,1$ & $\leq 0,01$ \\
Limitação por aspectos físicos & $80,2 \pm 28,5$ & $88,5 \pm 19,5$ & 0,12 \\
Dor & $68,2 \pm 16,9$ & $70,5 \pm 15,5$ & 0,65 \\
Estado geral de saúde & $54,9 \pm 11,2$ & $78,2 \pm 13,2$ & $\leq 0,01$ \\
Vitalidade & $50,8 \pm 11,6$ & $71,7 \pm 10,3$ & $\leq 0,01$ \\
Aspectos sociais & $85,7 \pm 13,9$ & $87,5 \pm 13,8$ & 0,70 \\
Limitação por aspectos emocionais & $86,3 \pm 17,0$ & $94,4 \pm 12,7$ & 0,09 \\
Saúde mental & $55,3 \pm 13,6$ & $72,3 \pm 17,3$ & $\leq 0,01$ \\
\hline
\end{tabular}

\section{Discussão}

Os resultados do presente estudo sugerem que as idosas apresentaram uma redução nos sintomas depressivos e da frequência de participantes em provável episódio depressivo bem como, uma melhora nos seguintes componentes do SF-36: vitalidade, capacidade funcional, estado geral da saúde e saúde mental após uma intervenção de exercícios de força prescritos através da percepção de esforço.

O uso da percepção de esforço para a prescrição do treinamento de força, em detrimento da não utilização de RMs no TF, se justifica por diversos fatores, dentre os quais, a preferência do sujeito pela prática de exercícios em intensidades de esforço mais baixas. Focht ${ }^{24}$ e GLASS e STANTON ${ }^{25}$ verificaram que, quando sujeitos jovens auto selecionavam a carga que julgassem "suficientes para uma sessão de força”, cargas baixas/moderadas ( $\approx 56 \%$, e entre $42 \mathrm{e}$ $57 \%$ de $1 \mathrm{RM}$, respectivamente) para as respectivas quantidades de repetiçóes $(\approx 9$ e 10 repetiçôes, respectivamente) eram escolhidas. Também, a adoção de cargas com altas intensidades pode representar um fator decisivo na baixa aderência e um alto 
índice de desistência aos programas de exercícios físicos ${ }^{26-27}$. Além disso, possivelmente a utilização de esforços máximos durante períodos prolongados de treinamento, poderia estar associada a um alto risco potencial de desenvolvimento da síndrome de "overtraining" e lesão por uso excessivo em sujeitos de aptidão física elevada ${ }^{28}$.

Estudos avaliando os efeitos do treinamento físico aeróbico em pacientes deprimidos mostram que intensidades auto-selecionadas possuem um efeito antidepressivo importante ${ }^{29}$. A percepção de esforço por sua vez, apesar de não ser diretamente auto-selecionada, ajusta a carga de acordo com o entendimento do participante em relação ao esforço realizado, portanto, pode tornar a sessão de exercício mais agradável e prazerosa ${ }^{30}$.

Os efeitos antidepressivos encontrados no presente estudo corroboram com estudos da literatura. Por exemplo, Pereira et al..$^{31}$ encontraram uma redução de 11\% na pontuaçáo da EDG-15 após 10 semanas de exercícios de força, com uma intensidade de $75 \%$ de $1 \mathrm{RM}$, enquanto que em nosso estudo a redução foi de $30,8 \%$. Essa diferença provavelmente se deve as diferentes versôes dos instrumentos utilizados (EDG$30 \times$ EDG-15), que contem diferentes numero de itens, pontuaçôes máximas, pontos de corte, itens e variabilidades diferentes, as diferenças das populaçóes, sendo a população do presente estudo cinco anos mais nova e dos protocolos de treino em relação a frequência semanal (duas versus três vezes por semana), duraçáo do estudo (12 versus 10 semanas) e a forma de prescrição da intensidade do exercício (pela percepção subjetiva $\mathrm{x}$ repetições máximas).

O efeito antidepressivo do exercício poderia estar associado ao aumento da liberação de neurotrofinas, em especial, do Fator Neurotrófico Derivado do Cérebro (BDNF). De acordo com estudos recentes, a realização do treinamento de força, estaria possivelmente resultando em uma série de alteraçôes fisiológicas e bioquímicas envolvidas com a liberação destes neurotransmissores, bem como, a ativação de receptores específicos ligados a neurogenese e dessa forma diminuindo a depressão $0^{31-32}$.

O presente estudo encontrou melhoras nas subescalas de vitalidade, capacidade funcional, estado geral da saúde e saúde mental. A melhora nessas subescalas corroboram outros estudos, em idosos que também encontraram tais efeitos ${ }^{33}$. Tal melhora por ser explicada através de modelos teóricos como o modelo proposto por McAuley e Morris $^{34}$. De acordo com McAuley e Morris ${ }^{34}$, a melhora da QVRS pode ocorrer por três vias: 1 )
Melhora do Funcionamento físico, que envolve aspectos relacionados às limitaçóes, "disabilities", aptidão-física e composiçáo corporal; 2) Melhora da Função cognitiva, que envolve aspectos como memória e atenção e 3) "Self-related function", que envolve aspectos como o afeto, a autoestima e a autoeficácia.

No presente estudo, duas das três vias foram verificadas. $\mathrm{O}$ presente estudo encontrou incrementos na força muscular (resultados previamente publicados $)^{18}$. Os incrementos percentuais na força máxima foram similares ao estudo de Bottaro et al. ${ }^{35}$ os quais encontraram um incremento médio de $26 \%$ após 10 semanas de intervenção em idosos. Os aumentos na força máxima encontrados no presente estudo corroboram que a utilizaçáo de cargas no treinamento de força pode ser realizada por meio de esforços submáximos, neste caso, prescritos pela percepção de esforço. No estudo de Folland et al. ${ }^{13}$, dois grupos realizaram o TF durante nove semanas no exercício extensão de joelhos. Foram realizadas no grupo de Repetições Máximas (RMs), quatro séries de 10 repetições $(\approx 75 \%$ de $1 \mathrm{RM})$, com intervalos de 30 segundos, enquanto no outro grupo, utilizouse 40 séries de uma repetiçáo $(75 \%$ de $1 \mathrm{RM})$ e intervalos de 30 segundos. Os grupos apresentaram similares ganhos na força dinâmica máxima (1 RM; 34 vs $40 \%$, respectivamente) e na força isométrica máxima (18 vs $15 \%$, respectivamente). Segundo o modelo de McAuley e Morris ${ }^{34}$ e McAuley et al. ${ }^{36}$, o aumento da força pode resultar em um aumento da funcionalidade e assim, contribuir para uma melhora em alguns domínios da QVRS.

No presente estudo, a segunda via (melhora da função cognitiva) não foi avaliada. No entanto, a literatura suporta ganhos em diversos aspectos cognitivos ${ }^{37-39}$. Desta forma, especula-se que a melhora de alguns aspectos cognitivos, como a memória e a função executiva, possam ter mediado a melhora da QVRS encontrada no presente estudo.

Por ultimo, foi encontrada uma diminuiçáo dos sintomas depressivos no presente estudo. A literatura suporta a existência de uma relação entre QVRS e sintomas depressivos, ou seja, quanto melhor a QVRS de um indivíduo, menos sintomas depressivos ele vai apresentar, e vice-versa ${ }^{40-43}$. E ainda, que a diminuição dos sintomas depressivos através do exercício pode explicar, ao menos em parte, a melhora na QVRS ${ }^{44}$. Dessa forma, o treinamento de força prescrito pela percepção do esforço foi capaz de diminuir os sintomas depressivos, sendo esta diminuição um dos potenciais mediadores desta relação. 
Com os presentes resultados concluímos que o treinamento de força baseado na percepção de esforço foi um método efetivo para uma redução nos sintomas de depressivos das participantes bem como para a melhora da QVRS nas seguintes subescalas do SF-36: vitalidade, capacidade funcional, estado geral da saúde e saúde mental em idosas. Como principal limitação do presente estudo apresentamos a ausência de um grupo controle ou de intervenção diferenciada para que pudéssemos conduzir a conclusóes mais consistentes.

\begin{abstract}
Strength training decreases the depressive symptoms and improves the health-related quality of life in older women

The objective of this study was to evaluate the effects of strength training, based on the perceived effort, on depressive symptoms and Health-Related Quality of Life (HROoL) of elderly women. Twenty four older women ( $65.6 \pm 4.5$ years), performed training exercise two times per week during 12 weeks. The intensity was prescribed using the BORG perceived effort scale. The Geriatric Depression Scale (EDG) was used to evaluate the depressive symptoms intensity. The HRQoL, was evaluated thought the Short Form-36 (SF-36). A significant decrease of depressive symptoms was found. The HROoL improved in some, but not all subscales. Improvements were found at functional capacity, general heath, vitality and mental health subscales. The strength training, based on perceived effort, was an effective method to decrease the depressive symptoms and improve HROoL on the functional capacity, general heath, vitality and mental health subscales of SF-36 on older women.
\end{abstract}

KeY WoRdS: Aged; Depression; Exercise; Quality of life; Resistance training; Women.

\title{
Referências
}

1. Izquierdo M, Hakkinen K, Ibanez J, et al. Effects of strength training on submaximal and maximal endurance performance capacity in middle-aged and older men. J Strength Cond Res. 2003;17:129-39.

2. Alexopoulos GS. Depression in the elderly. Lancet. 2005;365:1961-70.

3. Andrade L, Caraveo-Anduaga JJ, Berglund P, et al. The epidemiology of major depressive episodes: results from the International Consortium of Psychiatric Epidemiology (ICPE) Surveys. Int J Methods Psychiatr Res. 2003;12:3-21.

4. American Psychiatric Association. Diagnostic and statistical manual of mental disorders. 5th ed. Arlington: American Psychiatric Association; 2013.

5. Fleck MP, Chachamovich E, Trentini C. Development and validation of the Portuguese version of the WHOQOLOLD module. Rev Saúde Públ. 2006;40:785-91.

6. Bridle C, Spanjers K, Patel S, Atherton NM, Lamb SE. Effect of exercise on depression severity in older people: systematic review and meta-analysis of randomised controlled trials. Br J Psychiatry. 2012;201:180-5.

7. Katula JA, Rejeski WJ, Marsh AP. Enhancing quality of life in older adults: a comparison of muscular strength and power training. Health Qual Life Outcomes. 2008;6:45.

8. Stanton R, Happell BM. An exercise prescription primer for people with depression. Issues Ment Health Nurs. 2013;34:626-30.

9. Stanton R, Reaburn P. Exercise and the treatment of depression: a review of the exercise program variables. J Sci Med Sport. 2013;17:177-82.

10. Bean JF, Kiely DK, LaRose S, O’Neill E, Goldstein R, Frontera WR. Increased velocity exercise specific to task training versus the National Institute on Aging's strength training program: changes in limb power and mobility. J Gerontol A Biol Sci Med Sci. 2009;64:983-91.

11. Farinatti PTVA, B. F. C. Estudo de freqüência cardíaca, pressão arterial e duplo-produto em exercícios contra-resistência e aeróbio contínuo. Rev Bras Ativ Fís Saúde. 2000;5:5-16. 
12. Wilborn C. The effects of exercise intensity and body position on cardiovascular variables during resistance exercise. J Exerc Physiol Online. 2004;7:29-36.

13. Folland JP, Irish CS, Roberts JC, Tarr JE, Jones DA. Fatigue is not a necessary stimulus for strength gains during resistance training. Br J Sports Med. 2002;36:370-3.

14. Tiggemann CL, Dias CP, Noll M, Schoenell MCW, Kruel LFM. Envelhecimento e treinamento de potência: aspectos neuromusculares e funcionais. Rev Educ Fís/UEM. 2013;24:295-304.

15. Gaya AG, Silva MF, Moreira RB. Ciências do movimento humano: introdução à metodologia da pesquisa. Porto Alegre: Artmed; 2008.

16. Craig CL, Marshall AL, Sjostrom M, et al. International physical activity questionnaire: 12-country reliability and validity. Med Sci Sports Exerc. 2003;35:1381-95.

17. Borg G. Escalas de Borg para a dor e esforço percebido. São Paulo: Manole; 2000.

18. Tiggemann CL. Comparação entre métodos de determinação da carga e de velocidade de execução do treinamento de força nas adaptações neuromusculares e no desempenho de capacidades funcionais em mulheres idosas [tese]. Porto Alegre (RS): Universidade Federal do Rio Grande do Sul; 2013.

19. Gearhart RFJ, Goss FL, Lagally KM, Jakicic JM, Gallagher J, Robertson RJ. Standardized scaling procedures for rating perceived exertion during resistance exercise. J Strength Cond Res. 2001;15:320-5.

20. Tiggemann CL, Korzenowski AL, Brentano MA, Tartaruga MP, Alberton CL, Kruel LFM. Perceived exertion in different strength exercise loads in sedentary, active, and trained adults. J Strength Cond Res. 2010;24:2032-41

21. Brown LE, Weir JP. ASEP procedures recommendation i: accurate assessment of muscular strength and power. J Exerc Physiol Online. 2001;4:1-21.

22. Yesavage JA. Geriatric depression scale. Psychopharmacol Bull. 1988;24:709-11.

23. Ciconelli RM, Ferraz MB, Santos W, Meinao I, Quaresma MR. Tradução para a lingua portuguesa e validação do questionário genérico de avaliação da qualidade de vida SF-36. Rev Bras Reumatol. 1999;39:143-50.

24. Focht BC. Perceived exertion and training load during self-selected and imposed-intensity resistance exercise in untrained women. J Strength Cond Res. 2007;21:183-7.

25. Glass SC, Stanton DR. Self-selected resistance training intensity in novice weightlifters. J Strength Cond Res. 2004;18:324-7.

26. Perri MG, Anton SD, Durning PE, et al. Adherence to exercise prescriptions: effects of prescribing moderate versus higher levels of intensity and frequency. Health Psychol. 2002;21:452-8.

27. Lind E, Joens-Matre RR, Ekkekakis P. What intensity of physical activity do previously sedentary middle-aged women select? Evidence of a coherent pattern from physiological, perceptual, and affective markers. Prev Med. 2005;40:407-19.

28. Willardson JM. The application of training to failure in periodized multiple-set resistance exercise programs. J Strength Cond Res. 2007;21:628-31.

29. Callaghan P, Khalil E, Morres I, Carter T. Pragmatic randomised controlled trial of preferred intensity exercise in women living with depression. BMC Public Health. 2011;11:465.

30. Ekkekakis P, Hall E, Petruzzello S. The relationship between exercise intensity and affective responses demystified: to crack the 40-year-old nut, replace the 40-year-old nutcracker! Ann Behav Med. 2008;35:136-49.

31. Pereira DS, Queiroz BZ, Miranda AS, Rocha NP, et al. Effects of physical exercise on plasma levels of brain-derived neurotrophic factor and depressive symptoms in elderly women-a randomized clinical trial. Arch Phys Med Rehabil. 2013;94:1443-50.

32. Schuch FB, Almeida Fleck MP. Is Exercise an efficacious treatment for depression? A comment upon recent negative findings. Front Psychiatry. 2013;4:20.

33. Eyigor S, Karapolat H, Durmaz B. Effects of a group-based exercise program on the physical performance, muscle strength and quality of life in older women. Arch Gerontol Geriatrics. 2007;45:259-71.

34. McAuley E, Morris KS. State of the art review: advances in physical activity and mental health: quality of life. Am J Lifestyle Med. 2007;1:389-96.

35. Bottaro M, Machado S, Nogueira W, Scales R, Veloso J. Effect of high versus low-velocity resistance training on muscular fitness and functional performance in older men. Eur J Appl Physiol. 2007;99:257-64.

36. McAuley E, Doerksen SE, Morris KS, et al. Pathways from physical activity to quality of life in older women. Ann Behav Med. 2008;36:13-20.

37. Cassilhas RC, Viana VAR, Grassmann V, et al. The impact of resistance exercise on the cognitive function of the elderly. Med Sci Sports Exerc. 2007;39:1401-7.

38. Alves CRR, Merege Filho CAA, Benatti FB, et al. Creatine supplementation associated or not with strength training upon emotional and cognitive measures in older women: a randomized double-blind study. PLoS One. 2013;8:e76301. 
39. Peig-Chiello P, Perrig WJ, Ehrsam R, Staehelin HB, Krings F. The effects of resistance training on well-being and memory in elderly volunteers. Age Ageing. 1998;27:469-75.

40. Fleck MP, Lima AF, Louzada S, et al. associação entre sintomas depressivos e funcionamento social em cuidados primários à saúde. Rev Saude Publica. 2002;36:431-8.

41. Fleck MPA, Simon G, Herrman H, Bushnell D, Martin M, Patrick D. Major depression and its correlates in primary care settings in six countries: 9-month follow-up study. Brit J Psych. 2005;186:41-7.

42. Rocha N, Schuch F, Fleck M. Gender differences in perception of quality of life in adults with and without chronic health conditions: the role of depressive symptoms. J Health Psychol. 2013.

43. Rocha NS, Fleck MP. Evaluation of quality of life in adults with chronic health conditions: the role of depressive symptoms. Rev Bras Psiquiatria. 2010;32:119-24.

44. Schuch FB, Pinto SS, Bagatini NC, et al. Water-based exercise and quality of life in women: the role of depressive symptoms. Women Health. 2014;54:161-75.

\begin{tabular}{|c|c|}
\hline $\begin{array}{r}\text { ENDEREÇO } \\
\text { Felipe Barreto Schuch } \\
\text { Laboratório de Pesquisa do Exercício } \\
\text { Escola de Educação Física } \\
\text { Universidade Federal do Rio Grande do Sul } \\
\text { R. Felizardo, 750 - sala } 208 \\
\text { 90690-20o - Porto Alegre - RS - BRASIL } \\
\text { e-mail: felipe.schuch@ufrgs.br }\end{array}$ & $\begin{array}{l}\text { Recebido para publicação: 24/06/2014 } \\
\text { Revisado: 26/12/2014 } \\
\text { Aceito: 19/02/2015 }\end{array}$ \\
\hline
\end{tabular}

\section{Errata}

\section{v.29, n.2, abr./jun. 2015}

http://dx.doi.org/10.1590/1807-55092016000100217

No artigo "Treinamento de força diminui os sintomas depressivos e melhora a qualidade de vida relacionada a saúde em idosas", de autoria de Letícia Brunoni, Felipe Barreto Schuch, Caroline Pieta Dias, Luis Fernando Martins Kruel e Carlos Leandro Tiggeman, com número de DOI: http:// dx.doi.org/10.1590/1807-55092015000200189, publicado na Revista Brasileira de Educação Física e Esporte, v.29, n.2, abr./jun. 2015, p.189-96, onde se lia:

"Carlos Leandro Tiggeman"

Leia-se:

"Carlos Leandro Tiggemann". 